\title{
ON A NON-HOMOGENEOUS DIFFERENCE EQUATION FROM PROBABILITY THEORY
}

\author{
Jean-LuC Guilbault — Mario Lefebvre
}

\begin{abstract}
The so-called gambler's ruin problem in probability theory is considered for a Markov chain having transition probabilities depending on the current state. This problem leads to a non-homogeneous difference equation with non-constant coefficients for the expected duration of the game. This mathematical expectation is computed explicitly.
\end{abstract}

\section{Introduction}

We consider a Markov chain $\left\{X_{n}, n=0,1, \ldots\right\}$ having state space $S:=$ $\{0, \ldots, N\}$ and for which the (time-homogeneous) transition probabilities

$$
p_{i, j}:=P\left[X_{n+1}=j \mid X_{n}=i\right]
$$

are given by

$$
p_{i, i+1}=\frac{1}{4}(1-c i), \quad p_{i, i-1}=\frac{1}{4}(1+c i) \quad \text { and } \quad p_{i, i}=\frac{1}{2}
$$

for $i=1, \ldots, N-1$. The states 0 and $N$ are assumed to be absorbing, so that $p_{0, j}=\delta_{0, j}$ and $p_{N, j}=\delta_{N, j}$. Moreover, because $p_{i, j} \in[0,1]$ for all $i, j \in S$ and the state $N$ must be accessible from $N-1$, the positive constant $c$ must satisfy the condition

$$
c<\frac{1}{N-1} .
$$

Using the results in Cox and Miller (1965, p. 213), we can state that the Markov chain defined above may be regarded as a discrete version of the Ornstein-Uhlenbeck process. Various discrete versions of the Ornstein-Uhlenbeck process have been considered by, in particular, Renshaw (1997), A n is hchenko et al. (2002, p. 53), S prott (2003, p. 234), Larralde (2004 a,b), Kontoyiannis and Meyn (2005), and Milstein et al. (2007).

2000 Mathematics Subject Classification: 39A05, 60J10.

Keywords: Markov chain, ruin problem, Ornstein-Uhlenbeck process, non-constant coefficients, hypergeometric function, reducible equation.

Supported by the Natural Sciences and Engineering Research Council of Canada. 
Assume that $X_{0}=i \in\{1, \ldots, N-1\}$, and let $T:=\inf \left\{n>0: X_{n}=0\right.$ or $\left.N\right\}$. We want to determine the value of

$$
D_{i}:=E\left[T \mid X_{0}=i\right]
$$

The quantity $D_{i}$ is thus the expected value of the time (that is, the number of transitions) needed for the Markov chain to hit either 0 or $N$, starting from $i$. An application of this type of problem is the following: we can imagine that a gambler, having initially $i$ units of money, bets 1 unit at each play of a game for which he has a probability $p_{i, i+1}$ (respectively $p_{i, i-1}, p_{i, i}$ ) of winning (resp. losing, drawing). If $X_{T}=0$, we say that the gambler is ruined, hence the classical problem called the gambler's ruin problem. Here, $D_{i}$ is the expected duration of the game.

Conditioning on the outcome of the first play, we can write that $D_{i}$ satisfies the non-homogeneous difference equation with non-constant coefficients

$$
D_{i}=\frac{1-c i}{4} D_{i+1}+\frac{1+c i}{4} D_{i-1}+\frac{1}{2} D_{i}+1
$$

that is,

$$
\frac{[1-c(i+1)]}{2} D_{i+2}-D_{i+1}+\frac{[1-c(i+1)]}{2} D_{i}=-2 .
$$

The boundary conditions are

$$
D_{0}=D_{N}=0
$$

When $\left\{X_{n}, n=0,1, \ldots\right\}$ is a random walk, that is, when $p_{i, i+1}=p=1-p_{i, i-1}$ for $i=1, \ldots, N-1$, where $p \in(0,1)$, equation (4) becomes

$$
D_{i}=p D_{i+1}+(1-p) D_{i-1}+1 \text {. }
$$

It is not difficult to find the solution to this difference equation that satisfies $D_{0}=D_{N}=0$ (see for instance Felle r, 1968, p. 348). However, it turns out that solving (5), (6) is much more difficult. In Section 2, we will compute $D_{i}$ explicitly, and we will end this work with a few remarks in Section 3.

\section{Expected duration of the game}

In Lefebvre and Guilbault (2008), the authors calculated the first hitting place probability

$$
p_{i}:=P\left[X_{T}=N \mid X_{0}=i\right] .
$$

This probability is the solution of

$$
p_{i}=\frac{(1-c i)}{2} p_{i+1}+\frac{(1+c i)}{2} p_{i-1},
$$




\section{ON A NON-HOMOGENEOUS DIFFERENCE EQUATION FROM PROBABILITY THEORY}

for $i=1, \ldots, N-1$, that satisfies the boundary conditions $p_{0}=0$ and $p_{N}=1$. To obtain an explicit expression for $p_{i}$, the authors first set $x=i-1$ and let $y(x)=p_{i-1}$, so that equation (8) could be rewritten as

$$
\left[\frac{1-c(x+1)}{2}\right] y(x+2)-y(x+1)+\left[\frac{1+c(x+1)}{2}\right] y(x)=0
$$

for $x=0, \ldots, N-2$. The boundary conditions were $y(0)=0$ and $y(N)=$ 1. Notice that (9) is a hypergeometric difference equation (see B at c held er, 1967, p. 68).

Next, they transformed (9) into its normal form:

$$
(x-a) y(x+2)+a y(x+1)-x y(x)=0,
$$

where $a:=\frac{2}{c}$ and $x \in\left\{1+\frac{1}{c}, \ldots, N-1+\frac{1}{c}\right\}$. They found (see B a t c h eld er, 1967, Chapter III) that a fundamental system of solutions of equation (10) is $y_{1}(x) \equiv 1 \in \mathbb{R}$ and

$$
y_{2}(x)=(-1)^{x} \frac{\Gamma(x)}{\Gamma(x-a)} F\left(-a, 1, x-a, \frac{1}{2}\right),
$$

in which $F(\cdot, \cdot, \cdot, \cdot)$ is a hypergeometric function (see Abra mowitz and S t e g u n, 1965, p. 556).

Finally, they showed that the general solution of (10) can be expressed as

$$
y(x)=c_{1}+c_{2}(-1)^{[x]} \frac{\Gamma(x)}{\Gamma(x-a)} F\left(-a, 1, x-a, \frac{1}{2}\right),
$$

where [ ] denotes the integer part, and $c_{1}$ and $c_{2}$ are arbitrary constants.

\section{Remarks.}

i) The integer part of $x$ was used because the function $y(x)$ must be realvalued in the application considered. Moreover, the constants $c_{1}$ and $c_{2}$ are also assumed to be real.

ii) When the constant $c$ is of the form $c=1 /(N+j)$ or $c=2 /[2(N+j)-1]$, where $j \in\{0,1, \ldots\}$, equation (10) is completely reducible (see B a t c h e ld e r, 1967, p. 123-124). The case when $c=2 /[2(N+j)-1]$ does not cause any problem, but when $c=1 /(N+j)$ the function $y_{2}(x)$ can be expressed as

$$
y_{2}(x)=(-1)^{a+1} \frac{a !}{2^{a}} \quad \text { for } \quad x=1,2, \ldots, a+1 .
$$

Therefore, $y_{1}(x) \equiv 1$ and $y_{2}(x)$ are not linearly independent. However, $y_{2}(x)$ being a continuous function of the parameter $c$, the authors could take the limit as $c$ tends to $1 /(N+j)$ to obtain the solution for this value of $c$. 


\section{JEAN-LUC GUILBAULT — MARIO LEFEBVRE}

Now, with $x=i-1$ as above, and $z(x):=D_{i-1}$, the normal form of equation (5) is

$$
(x-a) z(x+2)+a z(x+1)-x z(x)=2 a .
$$

We already know the general solution of the corresponding homogeneous equation. Hence, we must find a particular solution of (13) to obtain its general solution. To do so, we will make use of a method that is similar to the method of variation of parameters for ordinary differential equations. We assume that the constant $c$ is different from $1 /(N+n)$. As mentioned above, the solution for this value of $c$ is obtained as a limit.

If $z_{1}(x)$ and $z_{2}(x)$ form a fundamental system of solutions for the associated homogeneous equation, and if $D(x)$ is the Casorati determinant, then a particular solution of (13) is (see B a t c held er, 1967, p. 13)

$$
z_{p}(x)=c_{1}(x) z_{1}(x)+c_{2}(x) z_{2}(x),
$$

where

$$
\Delta c_{n}(x)=\frac{M_{2 n}(x)}{D(x+1)} \frac{2 a}{(x-a)} \quad \text { for } \quad n=1,2
$$

and $M_{2 n}(x)$ is the cofactor of the second element in the $n$th column of

$$
D(x+1)=\left|\begin{array}{ll}
1 & z_{2}(x+1) \\
1 & z_{2}(x+2)
\end{array}\right|
$$

Hence,

and

$$
\Delta c_{1}(x)=-\frac{z_{2}(x+1)}{D(x+1)} \frac{2 a}{(x-a)}
$$

$$
\Delta c_{2}(x)=\frac{1}{D(x+1)} \frac{2 a}{(x-a)} .
$$

Remark. In the sequel, we assume that $\frac{\Gamma(z)}{\Gamma(w)}=\lim _{\epsilon \rightarrow 0} \frac{\Gamma(\epsilon+z)}{\Gamma(\epsilon+w)}$.

LEMMA 2.1. When $c-a \neq 0$ and $z \neq 0$,

$$
F(a, 1, c+1, z)=\frac{\Gamma(c+1)}{\Gamma(c)} \frac{\Gamma(c-a)}{\Gamma(c-a+1)} \frac{1}{z}\{1+(z-1) F(a, 1, c, z)\} .
$$

P r o of. The result follows directly from the following relation (see A b r a m owitz and Stegun, 1965, p. 558):

$$
c(1-z) F(a, b, c, z)-c F(a, b-1, c, z)+(c-a) z F(a, b, c+1, z)=0,
$$

and from the fact that $F(a, 0, c, z) \equiv 1$. 
Next, for equation (13) the Casorati determinant is

$$
\begin{aligned}
D(x)= & (-1)^{[x]+1} \frac{\Gamma(x+1)}{\Gamma(x+1-a)} F\left(-a, 1, x+1-a, \frac{1}{2}\right) \\
& -(-1)^{[x]} \frac{\Gamma(x)}{\Gamma(x-a)} F\left(-a, 1, x-a, \frac{1}{2}\right)
\end{aligned}
$$

and, making use of Lemma 2.1, we may write that

$$
D(x)=2(-1)^{[x]+1} \frac{\Gamma(x)}{\Gamma(x-a)} .
$$

Hence, the determinant is never equal to zero if $c \neq 1 /(N+n)$, with $n \in \mathbb{N}$. Furthermore, from equation (15),

$$
\Delta c_{2}(x)=(-1)^{[x]} a \frac{\Gamma(x-a)}{\Gamma(x+1)} .
$$

Remark. When $\frac{1}{c}=N+n-\frac{1}{2}, x$ is not an integer and the Casorati determinant is never equal to zero for all admissible values of $x$, as should be. In this case,

$$
\Delta c_{2}(x)=(-1)^{[x]} a \prod_{j=0}^{a}(x-j)^{-1} .
$$

However, if $\frac{1}{c}=N+n, D(x)$ is a polynomial of degree $a=2(N+n)$, which is zero over the set $S_{1}=\{1,2, \ldots, a\}$, but non-zero over $\mathbb{N} \backslash S_{1}$, that is, to the right of the singularity at $x=a$.

Now, an inverse difference $\Delta^{-1} f(x)$ of the function $f(x)$ is known every time either of the following formal series: $-\sum_{n=0}^{\infty} f(x+n), \sum_{n=1}^{\infty} f(x-n)$ converges (see B at chelder, 1967, p. 6). However, these series usually diverge. Nevertheless, they enable us to demonstrate the following proposition.

Proposition 2.1. Let

$$
f(x)=\eta^{x} \frac{\Gamma(x+\alpha)}{\Gamma(x+\beta)} .
$$

Then, everywhere the limit $\lim _{\epsilon \rightarrow 0} \frac{\Gamma(x+\epsilon+\alpha)}{\Gamma(x+\epsilon+\beta)}$ exists, we have

$$
\Delta^{-1} f(x)=-\eta^{x} \frac{\Gamma(x+\alpha)}{\Gamma(x+\beta)} F(x+\alpha, 1, x+\beta, \eta)
$$

or, equivalently,

$$
\Delta^{-1} f(x)=-\frac{\eta^{x}}{(1-\eta)} \frac{\Gamma(x+\alpha)}{\Gamma(x+\beta)} F\left[\beta-\alpha, 1, x+\beta, \frac{\eta}{(\eta-1)}\right],
$$

provided that $\eta<1$ or that $\beta-\alpha>1$ if $\eta=1$. In the case of the second equation, $\eta$ must be strictly smaller than 1 . 


\section{JEAN-LUC GUILBAULT — MARIO LEFEBVRE}

Pr o of. Making use of the formal series, we may write that

$$
\Delta^{-1} f(x)=-\eta^{x} \frac{\Gamma(x+\alpha)}{\Gamma(x+\beta)} F(x+\alpha, 1, x+\beta, \eta) .
$$

We can show (see Erdélyi et al., 1953, p. 68) that this function is entire when $|\eta|<1$. The series remains convergent when $\eta=1$ if $\beta-\alpha>1$. Moreover, by analytic continuation, this formula is valid for any $\eta<1$ and can then be written as in (17) (see A bramowitz and Steg un, 1965, p. 558), which is an entire function for any $\eta<1$.

Remark. When $\eta<0$, it is better to take the integer part of $x$ and write $\eta^{[x]}$ instead of $\eta^{x}$, in order to obtain only real values.

Next, from the previous proposition, if $c \neq 1 /(N+n)$, with $n \in \mathbb{N}$, we have

$$
c_{2}(x)=\frac{a}{2}(-1)^{[x]+1} \frac{\Gamma(x-a)}{\Gamma(x+1)} F\left(a+1,1, x+1, \frac{1}{2}\right) .
$$

Finally [see equation (11)],

$$
c_{2}(x) z_{2}(x)=-\frac{a}{2 x} F\left(a+1,1, x+1, \frac{1}{2}\right) F\left(-a, 1, x-a, \frac{1}{2}\right) .
$$

\section{Remarks.}

i) In the preceding equation, the second hypergeometric function is absolutely convergent when the state space of the Markov chain is $\{0,1, \ldots, N\}$, because $x-1>N-1>0$.

ii) In the particular case when $a=2(N+n)-1$, since $x-a$ is not a relative integer, we may write (see Abramowitz and Stegun, 1965, p. 561)

$$
c_{2}(x) z_{2}(x)=-\frac{a}{2 x} F\left(a+1,1, x+1, \frac{1}{2}\right) \sum_{n=0}^{a} \frac{(-a)_{n}}{2^{n}(x-a)_{n}} .
$$

The term $\Delta c_{1}(x)$ can be written as follows [see equation (14)]:

$$
\Delta c_{1}(x)=a F\left(-a, 1, x+1-a, \frac{1}{2}\right) \frac{\Gamma(x-a)}{\Gamma(x-a+1)} .
$$

In order to obtain an explicit expression for $c_{1}(x)$, we will use the Fundamental Theorem on summations.

Proposition 2.2. If $\Delta F(x)=f(x)$, and if $a, b(\geq a)$ are not integers, then

$$
\sum_{x=a}^{b} f(x)=\left.\Delta^{-1} f(x)\right|_{a} ^{b+1}=F(b+1)-F(a) .
$$


Now, since

$$
\Delta \sum_{t=a}^{x-1} f(t)=\Delta[F(x)-F(a)]=f(x),
$$

we have

$$
\Delta^{-1} f(x)=\sum_{t=a}^{x-1} f(t)
$$

(with the convention that $\sum_{t=a}^{a-1} f(t)=0$ ). Using this principle, equation (14) and the linearity of the inverse difference operator $\Delta^{-1}$, we deduce that

$$
\begin{aligned}
c_{1}(x) & =a \Delta^{-1}\left\{F\left(-a, 1, x+1-a, \frac{1}{2}\right) \frac{\Gamma(x-a)}{\Gamma(x-a+1)}\right\} \\
& =a \sum_{t=1+\frac{1}{c}}^{x-1} F\left(-a, 1, t+1-a, \frac{1}{2}\right) \frac{\Gamma(t-a)}{\Gamma(t-a+1)},
\end{aligned}
$$

where the summation $\sum_{t=1+\frac{1}{c}}^{x-1} f(t)$ is over $x-1-\frac{1}{c}$ elements. Furthermore, because $z_{1}(x) \equiv 1$, we have $c_{1}(x) z_{1}(x)=c_{1}(x)$, which is indeed a difference.

From the preceding results, we may state that a particular solution of equation (13) is given by

$$
\begin{aligned}
z_{p}(x)= & -\frac{a}{2 x} F\left(a+1,1, x+1, \frac{1}{2}\right) F\left(-a, 1, x-a, \frac{1}{2}\right) \\
& +a \sum_{t=1+\frac{1}{c}}^{x-1} F\left(-a, 1, t+1-a, \frac{1}{2}\right) \frac{\Gamma(t-a)}{\Gamma(t-a+1)} .
\end{aligned}
$$

This solution, evaluated at $x=1+\frac{a}{2}$, becomes (see Abramowitz and S t e g u n, 1965, p. 557)

$$
z_{p}\left[1+\left(\frac{a}{2}\right)\right]=\frac{a \pi}{2}\left\{\frac{\Gamma\left(1+\frac{a}{2}\right)}{\Gamma\left(\frac{1}{2}+\frac{a}{2}\right)}-\frac{1}{\sqrt{\pi}}\right\} \frac{\Gamma\left(\frac{-a}{2}\right)}{\Gamma\left(\frac{1}{2}-\frac{a}{2}\right)} .
$$

It follows that $z_{p}\left[1+\left(\frac{a}{2}\right)\right]=0$ when $c=2 /[2(N+n)-1]$, with $n \in \mathbb{N}$.

The general solution of equation (13), subject to the boundary conditions

$$
z\left[1+\left(\frac{a}{2}\right)\right]=z\left[N+1+\left(\frac{a}{2}\right)\right]=0,
$$

is now given in the following proposition.

Proposition 2.3. The average number of transitions $D_{i}$ defined in (3) is

$$
D_{i}=z\left[i+1+\left(\frac{1}{c}\right)\right]
$$


where

$$
z(x)=c_{1}+c_{2}(-1)^{[x]} \frac{\Gamma(x)}{\Gamma(x-a)} F\left(-a, 1, x-a, \frac{1}{2}\right)+z_{p}(x),
$$

$z_{p}(x)$ is given in (20),

$$
c_{2}=\frac{z_{p}\left[1+\left(\frac{a}{2}\right)\right]-z_{p}\left[N+1+\left(\frac{a}{2}\right)\right]}{z_{2}\left[N+1+\left(\frac{a}{2}\right)\right]-z_{2}\left[1+\left(\frac{a}{2}\right)\right]}
$$

and

$$
c_{1}=-c_{2} z_{2}\left[1+\left(\frac{a}{2}\right)\right]-z_{p}\left[1+\left(\frac{a}{2}\right)\right],
$$

in which the function $z_{2}(x)$ is the same as the function $y_{2}(x)$ in (11), but with $(-1)^{[x]}$ instead of $(-1)^{x}$.

In the particular case when

$$
c=\frac{2}{[2(N+n)-1]}, \quad \text { with } \quad n \in \mathbb{N},
$$

we have

$$
c_{1}=0 \quad \text { and } \quad c_{2}=\frac{-z_{p}\left[N+1+\left(\frac{a}{2}\right)\right]}{z_{2}\left[N+1+\left(\frac{a}{2}\right)\right]} .
$$

\section{Concluding remarks}

We have obtained an explicit expression for the average number $D_{i}$ of transitions needed for the Markov chain having the transition probabilities defined in (11) and starting at $X_{0}=i$ to hit either 0 or $N$. To do so, we solved the appropriate difference equation, subject to the boundary conditions $D_{0}=D_{N}=0$. We saw that the problem of finding a particular solution of (4) is not trivial.

Instead of proceeding as above, we could have solved a system of linear equations. This is straightforward when $N$ is small enough. However, in order to obtain an exact solution for any $N$, this technique is not convenient.

As in Lefebvre and Guilbault (2008), we could extend the problem solved in the current work by considering a Markov chain $\left\{X_{n}, n=0,1, \ldots\right\}$ with state space $\{-M, \ldots,-1,0,1 \ldots, N\}$, where $M \in\{1,2, \ldots\}$. Moreover, the probabilities $p_{i, j}$ could be different when $i$ is a negative state. That is, we could set

$p_{i, i+1}=\frac{1-d i}{4}, p_{i, i-1}=\frac{1+d i}{4} \quad$ and $\quad p_{i, i}=\frac{1}{2} \quad$ for $\quad i \in\{-M+1, \ldots,-1\}$, 


\section{ON A NON-HOMOGENEOUS DIFFERENCE EQUATION FROM PROBABILITY THEORY}

in which the constant $d(>0)$ must be such that $d<1 /(M-1)$ for the condition $0 \leq p_{i, j} \leq 1$ for all $i, j$ to be respected and the state $-M$ to be accessible from $-M+1$. When the process hits the origin, we could set

$$
p_{0,1}=p_{0}, p_{0,-1}=\frac{1}{2}-p_{0} \quad \text { and } \quad p_{0,0}=\frac{1}{2}, \quad \text { with } \quad p_{0} \in(0,1) .
$$

This type of Markov chain can have some interesting applications [see Lefebvre (2006)].

Finally, the fact that the Markov chain always has a $\frac{1}{2}$ probability of remaining in its current state does not influence the probability that it will hit 0 before $N$, for instance. However, it does of course increase the average number of transitions needed for the process to hit either 0 or $N$. We could also calculate the quantity $D_{i}$ when

$$
p_{i, i+1}=\frac{1-c i}{2} \quad \text { and } \quad p_{i, i-1}=\frac{1+c i}{2} \quad \text { for } \quad i \in\{1, \ldots, N-1\} .
$$

In this case, taking the limit as $c$ decreases to zero, we should retrieve the formula (see F eller, 1968, p. 349) $D_{i}=i(N-i)$.

\section{REFERENCES}

[1] ABRAmOWITZ, M.-STEGUN, I. A.: Handbook of Mathematical Functions with Formulas, Graphs, and Mathematical Tables. Dover, New York, 1965.

[2] ANISHCHENKO, V. S.-ASTAKHOV, V.-NEIMAN, A.-VADIVASOVA, T.-SCHIMANSKY-GEIER, L.: Nonlinear Dynamics of Chaotic and Stochastic Systems (2nd ed.), Springer-Verlag, Berlin, 2007.

[3] BATCHELDER, P. M.: An Introduction to Linear Difference Equations. Dover, New York, 1967.

[4] COX, D. R.-MILlER, H. D.: The Theory of Stochastic Processes. Methuen and Co. Ltd. X, London, 1965.

[5] ERdÉlyi, A.-MAGnus, W.-OBERHetTingeR, F.-TRICOMi, F. G.: Higher Transcendental Functions. Vol. I. Bateman Manuscript Project XXVI, McGraw-Hill Book Co., New York, 1953.

[6] FELLER, W.: An Introduction to Probability Theory and Its Applications, Vol. I (3rd ed.), Wiley, New York, 1968.

[7] KONTOYIANNIS, I.-MEYN, S. P.: Large deviations asymptotics and the spectral theory of multiplicatively regular Markov processes, Electron. J. Probab. 10 (2005), 61-123.

[8] LARRALDE, H.: A first passage time distribution for a discrete version of the Ornstein-Uhlenbeck process, J. Phys. A 37 (2004a), 3759-3767.

[9] LARRALDE, H.: Statistical properties of a discrete version of the Ornstein-Uhlenbeck process, Phys. Rev. E (3) 69 (2004b), 027102-4.

[10] LEFEBVRE, M.: First passage problems for asymmetric Wiener processes, J. Appl. Probab. 43 (2006), 175-184.

[11] LEFEBVRE, M.-GUILBAULT, J.-L.: First hitting place probabilities for a discrete version of the Ornstein-Uhlenbeck process (2008), (submitted for publication).

[12] Milstein, G. N.-SCHOENMAKERS, J. G. M.-SPOKOINY, V.: Forward and reverse representations for Markov chains, Stochastic Process. Appl. 117 (2007), 1052-1075. 


\section{JEAN-LUC GUILBAULT — MARIO LEFEBVRE}

[13] RENSHAW, E.: The discrete Uhlenbeck-Ornstein process, J. Appl. Probab. 24 (1987), 908-917.

[14] SPROTT, J. C.: Chaos and Time-Series Analysis. Oxford University Press, Oxford, 2003.

Received July 18, 2008

\author{
Département de mathématiques \\ et de génie industriel \\ École Polytechnique \\ C.P. 6079, Succursale Centre-ville \\ Montréal, Québec H3C $3 A 7$ \\ CANADA \\ E-mail: jean-luc.guilbault@polymtl.ca \\ mlefebvre@polymtl.ca
}

